
K. DÖRINGSHOFF ${ }^{1}$
I. ERNSTING ${ }^{1}$
R.-H. RINKLEFF ${ }^{2}$
S. SCHILLER ${ }^{1}$
A. WICHT $^{1, \infty}$

\title{
Frequency comb-referenced narrow line width diode laser system for coherent molecular spectroscopy
}

\author{
${ }^{1}$ Institut für Experimentalphysik, Heinrich-Heine-Universität Düsseldorf, Universitätsstr. 1, \\ 40225 Düsseldorf, Germany \\ 2 Institut für Gravitationsphysik, Leibniz Universität Hannover, Callinstr. 38, 30167 Hannover, Germany
}

\section{Received: 21 December 2007 \\ Published online: 28 February 2008 • C) Springer-Verlag 2008}

ABSTRACT We analyze in detail the frequency noise properties of a grating enhanced external cavity diode laser (GEECDL). This system merges two diode laser concepts, the grating stabilized diode laser and the diode laser with resonant optical feedback, thus combining a large tuning range with an excellent short-term frequency stability. We compare the frequency noise spectrum of a GEECDL to that of a grating stabilized diode laser and demonstrate a 10-fold reduction of the frequency noise linear spectral density. The GEECDL is phase locked to a similar laser and to a fs-frequency comb with a servo loop providing an open-loop unity-gain frequency of only $237 \mathrm{kHz}$, which is a tenth of the bandwidth typically required for grating stabilized diode lasers. We achieve a residual rms phase error as small as $72 \mathrm{mrad}(\approx 200 \mathrm{mrad}$ ) for stabilization to a similar laser (to the fs-frequency comb). We demonstrate that the novel diode laser can phase-coherently track a stable optical reference with an instability of $1.8 \times 10^{-16}$ at $1 \mathrm{~s}$. This laser system is well suited for applications that require phase locking to a low-power optical reference under noisy conditions. It may also be considered for the implementation of optical clock lasers.

PACS 42.55.Px; 42.60.Jf; 42.50.Gy

\section{Introduction}

Due to the recent progress in the fields of ultra-cold molecules and optical metrology the precise coherent manipulation of individual molecular rotational and vibrational quantum states has come into reach for experiments. In particular, new methods in trapping and cooling of molecular ions and the development of the fs-frequency comb provide the means to transfer theoretical concepts and experimental techniques from atom interferometry to a new field: molecular internal state quantum interferometry.

Due to the relatively large energy difference even between adjacent ro-vibrational quantum states of molecules, the coherent manipulation of individual molecular internal states requires multi-chromatic optical fields with ultra-stable difference frequencies in the range of a few $\mathrm{THz}$ to a few $10 \mathrm{THz}$. The most general way to provide these fields is to

Fax: +49-211-81-13116, E-mail: wicht@uni-duesseldorf.de phase lock multiple lasers, diode lasers in our case, to a fsfrequency comb that phase-coherently bridges the large frequency gaps [1-3]. However, phase locking of grating stabilized diode lasers to a fs-frequency comb is challenging, especially if small residual phase errors have to be guaranteed. This is due to the poor signal-to-noise ratio of the corresponding beat-note signal: the optical power contained in the individual modes of the fs-frequency comb is small $(\sim 10 \mathrm{nW})$ and the frequency noise bandwidth of grating stabilized diode lasers is large. The large noise bandwidth necessitates a control bandwidth of a few $\mathrm{MHz}$ for phase locking [4-8], and this implies that detection noise contained within a large bandwidth is fed into the servo loop. Our approach therefore is to develop diode lasers that feature reduced frequency noise, so that they can be phase locked to a fs-frequency comb with a servo bandwidth of only a fraction of $1 \mathrm{MHz}$. Because of their excellent short-term frequency stability, these diode lasers can also be considered for the implementation of local oscillators for optical clocks.

The aim of this paper is to discuss in detail the frequency noise properties of a grating enhanced external cavity diode laser (GEECDL) system $[9,10]$. To this end we determine the frequency noise spectral density of a free-running GEECDL and compare it to that of a Littman-type grating stabilized diode laser. We find a rms frequency jitter of $30 \mathrm{kHz}$ for the GEECDL, which is almost one order of magnitude smaller than the $208-\mathrm{kHz}$ jitter of the Littman diode laser. We further phase lock the GEECDL to a similar laser as well as to a fs-frequency comb and determine the corresponding residual phase noise linear spectral density. We also analyze the rf beat-note spectra, from which the fractional rf power contained in the coherent part of the beat-note signal can be determined. From the latter and from the phase noise spectra we can independently derive two values for the residual rms phase error of the optical phase-locked loops (OPLLs) and cross-check them for consistency. The phase lock between two GEECDLs yields a residual rms phase error of only $72 \mathrm{mrad}$ for a $10 \mathrm{~Hz}$ to $10 \mathrm{MHz}$ noise integration bandwidth. When phase locking a GEECDL to a fs-frequency comb we find a residual phase error of $194 \mathrm{mrad}$ for a $10 \mathrm{~Hz}$ to $500 \mathrm{kHz}$ integration bandwidth. Both results are achieved with a servo bandwidth that is one order of magnitude smaller than what is typically required to phase lock grating stabilized diode lasers to this level of instability $[1,2,4-8]$. This constitutes a ma- 
jor advancement for applications that require high-power, well-tuneable, ultra-low-noise or even phase-stabilized diode lasers.

The paper is organized as follows. We first introduce the diode laser setup and emphasize the novel aspects of the latest GEECDL version in Sect. 2. Then, in Sect. 3, we describe the experimental setup used to phase or frequency lock a GEECDL to a similar laser or to a fs-frequency comb. The setup is further employed to determine the frequency noise spectrum of a GEECDL and of a grating stabilized diode laser. The corresponding results are discussed in Sect. 4. We finally apply the setup to investigate the phase noise of a GEECDL that is phase locked either to another GEECDL or to a fsfrequency comb. This is described in Sect. 5. We conclude the discussion in Sect. 6.

\section{The latest version of the GEECDL}

The schematic setup of the grating enhanced external cavity diode laser (GEECDL) system is shown in Fig. 1. It can be considered a Littman configuration in which the retroreflecting mirror is replaced by a folded, confocal cavity. The basic concept underlying this diode laser system has been described in detail in [9]. Here, we restrict the discussion to a brief description of the experimental setup.

The optical output of the laser diode is collimated by an aspheric lens (Geltech 350390, $f=2.75 \mathrm{~mm}, \mathrm{NA}=0.65$ ) and is then diffracted by a volume holographic transmission grating (Wasatch Photonics, 980 1/mm, 95\% diffraction efficiency). The first diffraction order is coupled into a 75-mm-long external cavity with a folded confocal geometry. This cavity provides resonant optical feedback to the laser diode. It consists of two highly reflecting mirrors $\mathrm{MP}$ and $\mathrm{MF}(R=99.7 \%)$ and a plane mirror (transmission $T \approx 8.2 \%$ ) that provides input and output coupling. Because the optical setup constitutes two coupled cavities that are defined by (a) the rear facet of the laser diode chip and the mirror MF and (b) the mirrors MP and MF, stable single-mode operation of the diode laser requires control of the detuning between these cavities. To this end we apply a polarization-sensitive stabilization scheme originally

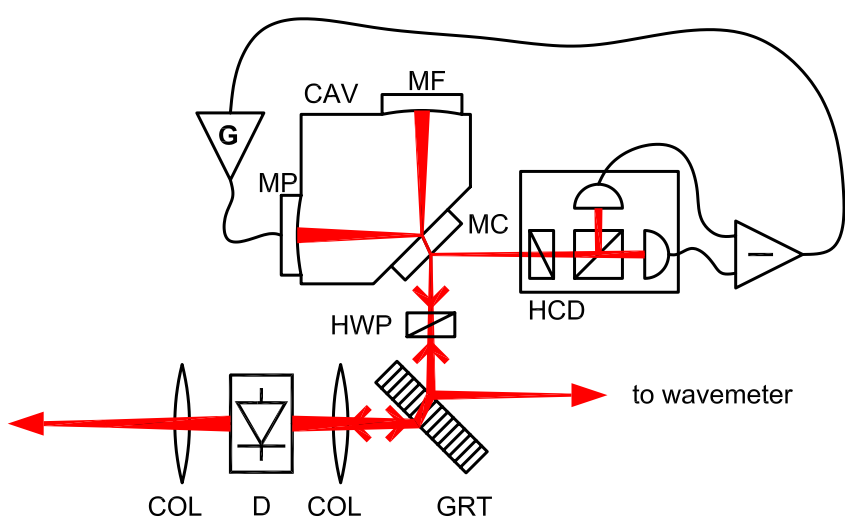

FIGURE 1 Schematic setup of the grating enhanced external cavity diode laser (GEECDL). D: laser diode, COL: collimator, GRT: transmission grating, HWP: half-wave plate, MC: coupling mirror, MF: mirror for frequency control, MP: mirror for phase control, CAV: external cavity, HCD: detector for relative frequency stabilization between both coupled cavities by means of a polarization-sensitive scheme, G: feedback electronics proposed by Hänsch and Couillaud [11]. With laser systems operating at $1400 \mathrm{~nm}$ we have recently demonstrated a large overall tuneability in excess of $50 \mathrm{~nm}$, a continuous tuneability of up to $20 \mathrm{GHz}$ and a short-term line width of only $11 \mathrm{kHz}$. Further, to emphasize the excellent short-term stability provided by this diode laser concept, we phase locked two similar lasers by means of a servo loop that used an analog phase detector and featured an open-loop unity-gain frequency as small as $46 \mathrm{kHz}$ [10].

In contrast to the low-power GEECDL described in [10] (GEECDL I for short), the optical output of the new GEECDL (GEECDL II for short) is provided through the rear facet of the laser diode chip (Sacher Lasertechnik SAL-1430100) [12]. This modifies the output coupling concept and thus increases output power, and it also allows for stable singlemode operation at large injection currents, which enhances the output power even further. According to the manufacturer, this new laser diode chip features a maximum injection current of $500 \mathrm{~mA}$ and provides stable single-mode operation in a grating laser configuration for injection currents up to $400 \mathrm{~mA}$. At this injection current it delivers an output power of approximately $100 \mathrm{~mW}$ at $1417 \mathrm{~nm}$. With our GEECDL setup we achieve single-mode operation at injection currents up to $400 \mathrm{~mA}$ as well, where the laser delivers an output power of $87 \mathrm{~mW}$ at $1420 \mathrm{~nm}$. This already includes the insertion loss of $1-T=24.7 \%$ introduced by an Isowave I-14 series double-stage optical isolator. We therefore conclude that the GEECDL system provides at least the same performance concerning single-mode operation at high injection currents and output power as regular grating stabilized diode lasers.

For comparison we may consider the earlier GEECDL I. This version provides stable single-mode operation in the GEECDL configuration up to $60 \mathrm{~mA}$, which corresponds to only $50 \%$ of the maximum rated injection current. It further delivers slightly less than $1 \mathrm{~mW}$ of optical power from a laser diode chip that nominally provides $10 \mathrm{~mW}$ of output power in a conventional grating stabilized setup. The 100-fold enhancement of output power yielded when switching from GEECDL I to GEECDL II is therefore in part (10-fold) due to the modification of the output coupling concept. We therefore conclude that a laser geometry which generates laser output from one facet of the chip and allows for optical feedback from an external cavity through the other facet is the optimum geometry for the implementation of the GEECDL concept. The latest version of the GEECDL now provides sufficient power for the implementation of an OPLL to a fs-frequency comb and for coherent molecular spectroscopy.

3

\section{Noise analysis setup}

We now describe the experimental setup which is used to frequency or phase lock GEECDL II to GEECDL I or to a fs-frequency comb. This setup is also used to analyze the frequency and phase noise spectra of a GEECDL and of a Littman-type grating stabilized diode laser.

In order to determine the frequency noise spectrum of the beat-note signal between GEECDL II and another laser, the corresponding laser beams are overlapped by means of a spliced single-mode optical fiber; see Fig. 2. Two copies of the beat note are then detected with two fiber-coupled pho- 


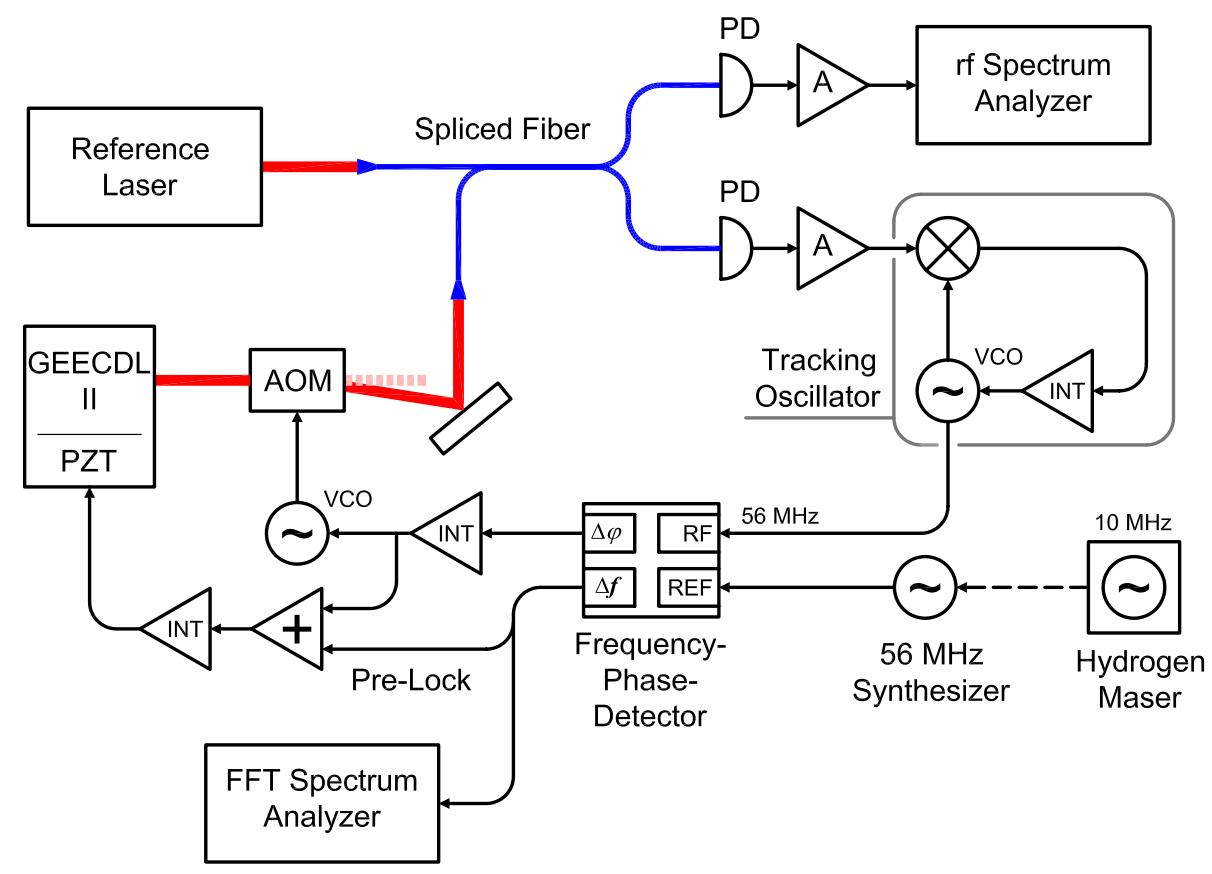

FIGURE 2 Schematic of the OPLL and of the frequency and phase noise analysis setup. The beat note between two lasers is recorded with two identical photodetectors. The output of one of the detectors is used for rf signal analysis of the beat-note signal, whereas the signal of the other is actively filtered by a tracking oscillator. The filtered beat-note signal is then analyzed by a frequency-phase detector. The latter provides an error signal for relative frequency stabilization between the two lasers at the output $\Delta f$. The output $\Delta f$ can also be used to determine the frequency noise spectrum of the filtered beat note. Additionally, the output $\Delta \varphi$ can be used to create an error signal for phase locking the beat-note signal to a stable rf reference

todetectors (Thorlabs D400FC). The output signal of one of those is used for rf signal analysis with an Agilent E4440A spectrum analyzer, whereas the output signal of the other is actively filtered by a fast tracking oscillator with $2-\mathrm{MHz}$ servo bandwidth. The filtered beat-note signal is then delivered to a combined frequency-phase detector [13]. Its frequency-tovoltage converter part provides a modulation detection bandwidth of $10 \mathrm{MHz}$. The corresponding output $\Delta f$ is delivered to a fast Fourier transform (FFT) spectrum analyzer (Stanford Research Systems SRS 780) to record the frequency noise linear spectral density (LSD) of the beat-note signal. This output is also used to derive an error signal for the implementation of a relative frequency stabilization between GEECDL II and the other laser by actuating the piezo-electric transducer (PZT) that drives the mirror MF of GEECDL II.

To phase lock GEECDL II to an optical reference we make use of the phase-detector part of the frequency-phase detector; see Fig. 2. The corresponding detector output $\Delta \varphi$ provides a purely analog, linearized measurement of the phase difference between the filtered beat-note signal and a rf reference signal, which has a frequency of approximately $56 \mathrm{MHz}$ and is provided by a rf synthesizer (Hewlett-Packard 8657B). The optical phase of GEECDL II is controlled by means of an acousto-optic modulator (AOM) for Fourier frequencies up to $500 \mathrm{kHz}$, and by means of a PZT acting on mirror MF of GECCDL II to control drifts and low-frequency jitter for Fourier frequencies up to approximately $50 \mathrm{~Hz}$.

To characterize the OPLL servo properties we determine its open-loop transfer function from a closed-loop measurement. This reveals an open-loop unity-gain frequency (loop bandwidth for short) of approximately $237 \mathrm{kHz}$ for the phase lock to both another GEECDL and to the fsfrequency comb. This is reflected by servo bumps found in the beat-note spectra at a carrier offset frequency of $290 \mathrm{kHz}$ and $260 \mathrm{kHz}$; see Figs. 6 and 7, respectively. We point out that in many papers the servo bandwidth is cited and is considered to be given by the servo-bump frequency itself. However, we feel that this is too rough an approximation, as the servo bandwidth, i.e. the frequency at which the closed-loop transfer function rolls off by $-3 \mathrm{~dB}$, is typically significantly larger - by a factor of two or so - than the servo-bump frequency or the open-loop unity-gain frequency. Because other papers typically give servo-bump frequencies, we prefer to refer to the servo-bump frequency or loop bandwidth rather than to the servo bandwidth for comparison.

\section{$4 \quad$ Frequency noise analysis}

In order to characterize the frequency noise of the free-running GEECDL we take a beat note between GEECDL I and GEECDL II, both free-running, and determine the frequency noise LSD of the beat-note signal. This sets an upper limit to the frequency noise spectrum of the free-running GEECDL. We are also interested in comparing the noise properties of a GEECDL to that of a regular grating diode laser in Littman configuration. To this end we take a beat note between GEECDL II and GEECDL I after the latter was 'downgraded' to a regular Littman laser by replacing the external confocal cavity by a plane dielectric mirror $(R>99 \%)$. This strategy ensures that the differences between the frequency noise spectra are solely due to the difference between the feedback concepts. Because, as we will see, GEECDL II is more frequency-stable than the regular grating diode laser, the frequency noise spectrum of the corresponding beat note describes the typical frequency noise spectrum of a Littman-type diode laser.

To determine the sensitivity of our setup for frequency noise measurements, we use a synthesizer to mimic a quasinoiseless filtered beat-note signal and analyze it at the output $\Delta f$ of the frequency-to-voltage converter with the FFT spectrum analyzer. The result is depicted as graph (A) in Fig. 3. Alternatively, we also phase lock the beat-note signal between two GEECDLs to a stable rf reference and use the correspond- 


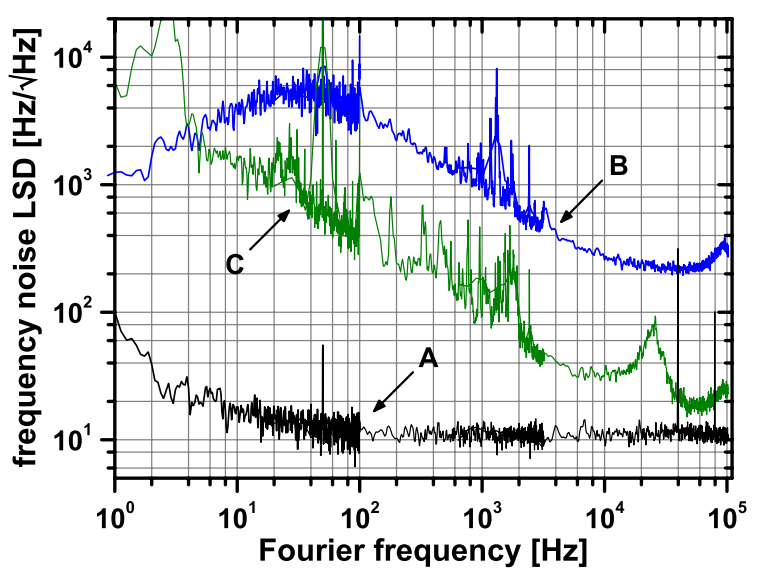

FIGURE 3 Frequency noise linear spectral density of the rf signals corresponding to the beat notes between (B) a diode laser in Littman configuration and a GEECDL that is frequency locked to the Littman laser with a loop bandwidth of approximately $50 \mathrm{~Hz}$ and (C) two GEECDLs, both free running. Graph (A) depicts the detector noise floor

ing highly frequency-stable signal to analyze the sensitivity of our setup. The result is basically identical to curve (A).

Graph (B) of Fig. 3 shows the result of a beat-note measurement between GEECDL II and the Littman laser. To eliminate the frequency drift between the lasers we frequency lock GEECDL II to the Littman laser with a loop bandwidth of approximately $50 \mathrm{~Hz}$. Above $50 \mathrm{~Hz}$ the frequency noise LSD equals that of the free-running lasers, dropping down to approximately $300 \mathrm{~Hz} / \sqrt{\mathrm{Hz}}$ at $100 \mathrm{kHz}$. The $f^{-1 / 2}$ sloping background is attributed to injection-current noise. The spikes around $1 \mathrm{kHz}$ indicate mechanical resonances of the setup. Please note that below $50 \mathrm{~Hz}$ the frequency noise is suppressed by the relative frequency lock between the two lasers. A comparison to recent results of other groups $[14,15]$ indicates that the frequency noise spectrum found here is typical for that of well-built grating stabilized diode lasers.

From the data shown in Fig. 3 we calculate the rms frequency jitter $\Delta v_{\text {jitter }}$ of the beat-note signal by integrating the frequency noise spectrum according to the relation

$$
\Delta v_{\mathrm{jitter}}(f)=\sqrt{\int_{f_{0}}^{f}\left[S_{\mathrm{f}}(f)\right]^{2} \mathrm{~d} f},
$$

where $S_{\mathrm{f}}(f)$ is the frequency noise LSD of Fig. 3. The frequency jitter is shown as a function of the integration bandwidth $f$ in Fig. $4\left(f_{0}=10 \mathrm{~Hz}\right)$. For the beat-note measurement between the GEECDL and the Littman laser we obtain a rms frequency jitter of about $208 \mathrm{kHz}$ for a $100-\mathrm{kHz}$ integration bandwidth (graph (A)). This result is consistent with the beat-note spectrum (B) shown in Fig. 5 which was recorded with the same setup as the data shown in Fig. 3: averaging over 1000 sweeps (not shown) reveals a Lorentzian spectrum with a half-width at half-maximum of $205 \mathrm{kHz}$.

We next turn to the analysis of the GEECDL frequency noise, which is obtained from a beat-note measurement between GEECDL II and GEECDL I. The excellent passive stability of the GEECDLs allows us to operate both lasers free running. We tune them to a difference frequency of approximately $56 \mathrm{MHz}$, the nominal working frequency of

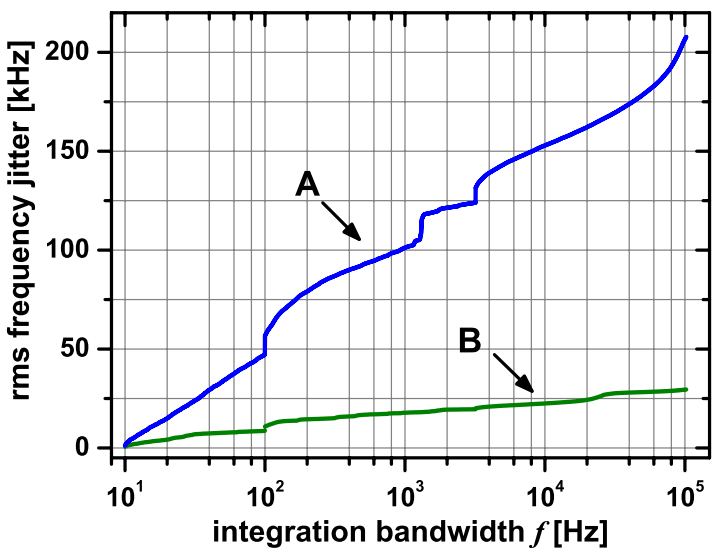

FIGURE 4 The rms frequency jitter according to (1) vs. integration bandwidth $f$ for the beat-note measurement (A) between GEECDL II and a diode laser in Littman configuration and (B) between GEECDLs I and II

the frequency-to-voltage converter, and record the frequency noise spectrum of the beat-note signal as described in Sect. 3 . We verify that the beat-note frequency remains close to the working frequency of the converter during the measurement. The result of this measurement is shown as graph (C) in Fig. 3. Comparison to graph (B), i.e. to the frequency noise spectrum of the Littman laser, indicates that the GEECDL concept reduces the frequency noise linear spectral density by one order of magnitude over the noise bandwidth recorded here. This measurement, for the first time, proves in detail the validity of the GEECDL concept. The noise suppression particularly at high Fourier frequency plays an important role for phase stabilization of these diode lasers, as already demonstrated [10]. It should be noted that further noise reduction should be feasible by increasing the external cavity finesse from its current value of approximately 38 to a few hundred. We finally point out that the GEECDL noise spectrum, in contrast to the Littman laser noise spectrum, reveals resonances at $1.6 \mathrm{kHz}$ and $26 \mathrm{kHz}$, which are attributed to resonances of the external cavity mechanical setup.

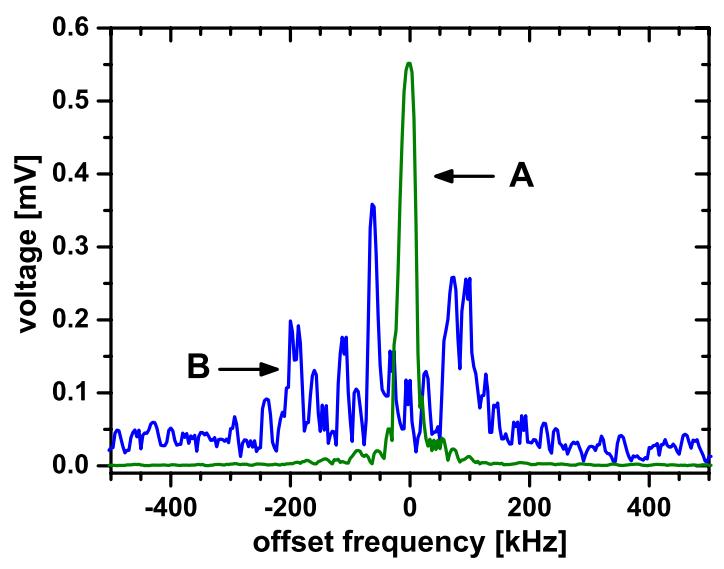

FIGURE 5 Single-sweep spectra of the rf signals corresponding to the beat notes between (A) two unstabilized GEECDLs and (B) a GEECDL and a diode laser in Littman configuration frequency locked to each other with a loop bandwidth of approximately $50 \mathrm{~Hz}$. Beat-note spectrum (A) shows a FWHM line width of $30 \mathrm{kHz}$ while (B) shows multiple peaks due to the relatively large frequency jitter of the Littman laser. Spectrum analyzer settings are $18-\mathrm{kHz}$ resolution bandwidth and 7.48-ms sweep time 
The rms frequency jitter of the beat-note signal between the two GEECDLs is depicted as graph (B) in Fig. 4, which indicates a value of only $30 \mathrm{kHz}$ for a $100-\mathrm{kHz}$ integration bandwidth. This is a reduction of almost one order of magnitude with respect to the frequency jitter of the Littman laser. This improvement is also reflected by the beat-note spectra shown in Fig. 5, where graph (A) corresponds to the spectrum of the beat note between two GEECDLs, both free running, and graph (B) refers to the beat-note spectrum between GEECDL II and the Littman laser, both locked to each other with a loop bandwidth of approximately $50 \mathrm{~Hz}$.

\section{5}

\section{Phase noise analysis}

The coherent manipulation of individual ro-vibrational states of molecules requires a stable, low-noise phase stabilization of diode lasers to a fs-frequency comb ${ }^{1}$. In Sect. 4 we showed that the GEECDL configuration efficiently reduces the frequency noise with respect to a standard grating stabilized diode laser. In this section we demonstrate that the noise reduction allows for a stable, low-noise phase stabilization to another GEECDL or to a fs-frequency comb with a purely analog phase-detection scheme and a loop bandwidth of only $237 \mathrm{kHz}$.

In order to give a quantitative characterization of the residual noise of the OPLLs we investigate the phase noise LSD of the beat-note signals and determine the fractional power contained in the coherent part of these signals. To characterize the long-term (in)stability of the phase stabilization we determine the corresponding Allan deviation.

\subsection{Fractional power contained in the carrier}

A good measure of the performance of an OPLL is the fractional power

$\eta=\frac{P_{\mathrm{C}}}{P_{\text {Noise }}}$

contained in the coherent part of the beat-note signal, i.e. in the 'carrier'. Accordingly, $P_{\mathrm{C}}$ denotes the coherent rf signal power and $P_{\text {Noise }}$ corresponds to the power of the rf noise contained within a certain integration bandwidth.

We first determine $\eta$ for a phase lock between two GEECDLs. To this end we phase lock GEECDL II to GEECDL I with a loop bandwidth of $237 \mathrm{kHz}$. We capture the beat-note spectrum with the rf spectrum analyzer for various span settings to ensure high spectral resolution close to the carrier.

The various spectra are then combined, as shown in Fig. 6, and their incoherent (noise) part is integrated over the noise window, which has a width of $20 \mathrm{MHz}$ and is centered at the carrier frequency. We find a fraction of $\eta=99.47 \%$ of the rf power to be contained in the carrier. This is a remarkable result given a loop bandwidth (open-loop unity-gain frequency) of only $237 \mathrm{kHz}$. In comparison, Cacciapuoti et al. [5] reported

\footnotetext{
${ }^{1}$ The transfer oscillator concept introduced by Telle et al. [16] allows us to eliminate phase noise of the fs-frequency comb from the measurement of the relative phase between two diode lasers that are each phase locked to the fs-frequency comb.
}

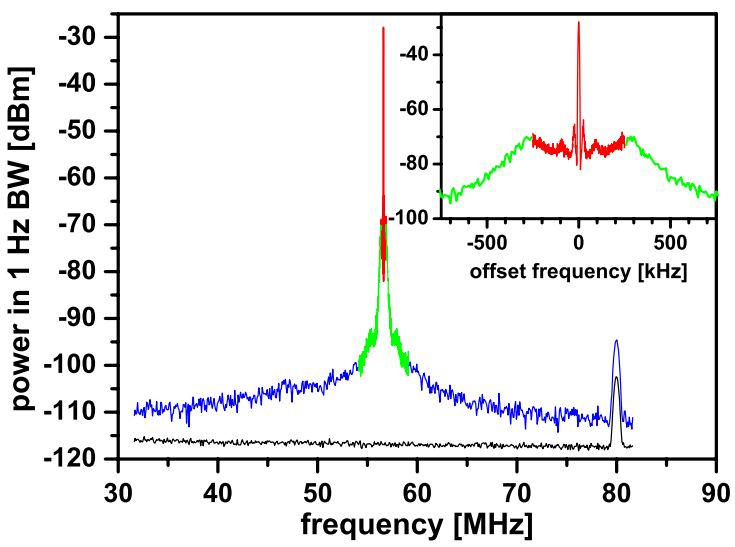

FIGURE 6 Power spectral density of the rf signal corresponding to the beat note between two similar GEECDLs phase locked to each other. The flat background (black graph) denotes the shot-noise level. The peak at $80 \mathrm{MHz}$ is attributed to rf pick-up from the AOM driving signal. The fractional power contained in the rf carrier signal at $56.09 \mathrm{MHz}$ is $99.47 \%$. The inset shows the beat-note spectrum at sub-MHz carrier offset frequencies. Servo bumps at $290 \mathrm{kHz}$ are clearly visible $(\mathrm{BW}=$ bandwidth $)$

a fractional power of $\eta=98.5 \%$ to be contained in the coherent signal when integrating the noise between $5 \mathrm{~Hz}$ and $10 \mathrm{MHz}$. In that work two Littrow lasers were phase locked to each other with a combination of analog and digital phase detection. The corresponding beat-note spectrum showed servo bumps at a carrier offset frequency of $3 \mathrm{MHz}$, from which we infer a loop bandwidth as large as $3 \mathrm{MHz}$.

Next, GEECDL II is phase locked to a fs-optical frequency comb (MenloSystems FC 8004) that is based on a Ti:sapphire oscillator (Femtolasers Femtosource Scientific 200) and spectral broadening with a photonic crystal fiber (Crystal Fibre Femtowhite 800). The fiber output covers a spectrum of approximately $500 \mathrm{~nm}$ to $1600 \mathrm{~nm}$. The carrier envelope offset frequency as well as the repetition rate are phase locked to $\mathrm{H}$-maser referenced synthesizers. The spectrum of the rf signal corresponding to the beat note between GEECDL II and the fs-frequency comb is recorded and composed as shown in Fig. 7. Now, the measurement sensitivity is limited by shot

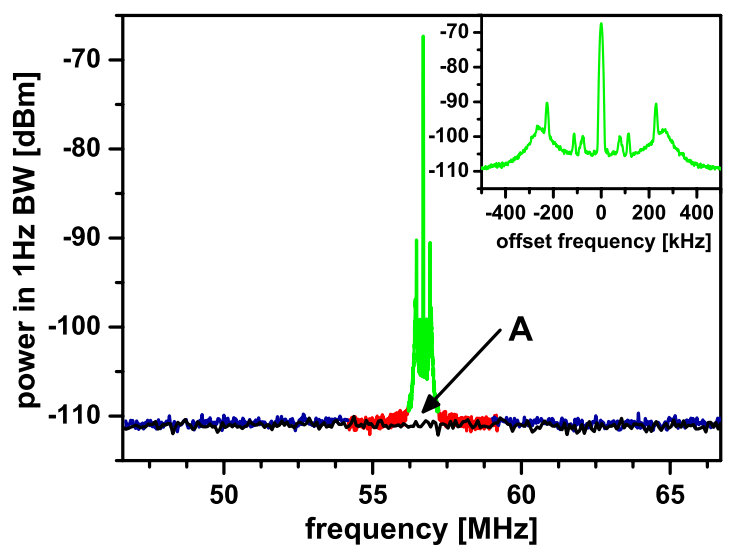

FIGURE 7 Power spectral density of the rf signal corresponding to the beat note between GEECDL II and the fs-frequency comb. The GEECDL is phase locked to the fs-frequency comb. The fractional power in the carrier at $56.70 \mathrm{MHz}$ is estimated to be $86.77 \%<\eta<96.31 \%$. The flat background (black graph) denotes the shot-noise level. The inset shows the beat-note spectrum with servo bumps at $260 \mathrm{kHz}(\mathrm{BW}=$ bandwidth $)$ 
noise of the diode laser, which is shown as graph (A). The beat-note spectrum hence contains phase noise information only in a bandwidth of approximately $\pm 500 \mathrm{kHz}$ around the carrier. We therefore can only determine upper and lower bounds to the fractional power contained in the carrier. Integration of the rf spectra over a 1-MHz-wide window centered at the carrier frequency yields a fractional power of $\eta=96.31 \%$ as an upper bound; integration over a $20-\mathrm{MHz}-$ wide window yields $\eta=86.8 \%$ as a lower bound. In fact, this is a remarkable result, given the loop bandwidth of only $237 \mathrm{kHz}$. For comparison, Yamazaki et al. [1] reported a fractional power of $\eta=80.4 \%$ to be contained in the carrier when they phase locked a grating laser to a fs-frequency comb. Here, noise was integrated over a $10-\mathrm{MHz}$ window, and the loop bandwidth, as estimated from the servo bump carrier offset frequency, was $2.3 \mathrm{MHz}$.

\subsection{Phase noise}

In the following we investigate the phase noise spectra of the rf signals that are derived from a beat-note measurement between phase-locked lasers.

We first phase lock GEECDL II to GEECDL I. The phase noise LSD of the beat-note signal is recorded with the phase noise utility of the Agilent E4440 A spectrum analyzer with the setup of Fig. 2. The result of this measurement is shown as graph (A) in Fig. 8. We recognize resonances at $1.6 \mathrm{kHz}$ and $26 \mathrm{kHz}$ that we already encountered when analyzing the frequency noise spectra. The OPLL servo bump is located at $250 \mathrm{kHz}$. Above $500 \mathrm{kHz}$ the phase noise LSD rolls off.

From this measurement the rms phase error $\varphi_{\text {rms }}$ can be evaluated according to

$\varphi_{\mathrm{rms}}^{2}=\int_{f_{0}}^{f}\left[S_{\varphi}(f)\right]^{2} \mathrm{~d} f$,

where $S_{\varphi}(f)$ is the single-sideband phase noise LSD. The fractional power contained in the carrier $\eta$ is then given by [8]

$\eta=\exp \left[-\varphi_{\mathrm{rms}}^{2}\right]$.

We calculate the rms phase error as a function of the integration bandwidth $\left(f_{0}=10 \mathrm{~Hz}\right)$. The result is shown as graph (A) in Fig. 9 and indicates that at $500 \mathrm{kHz}$ the rms phase error levels off at a value of $\varphi_{\mathrm{rms}}=72 \mathrm{mrad}$. From (2) we derive a fractional power of $99.48 \%$ to be contained in the carrier, which agrees very well with the $99.47 \%$ derived earlier from the rf beat-note spectrum; see Fig. 6 . Hence, with a loop bandwidth as small as $237 \mathrm{kHz}$ a rms phase error of significantly less than $100 \mathrm{mrad}$ is achieved, which renders cycle slips very unlikely for reasonable time scales [16]. We may compare this result to the result of Cacciapuoti et al. [5]. They employed a loop bandwidth as large as $3 \mathrm{MHz}$ to achieve a residual rms phase error of $100 \mathrm{mrad}$ in a $5-\mathrm{Hz}$ to $10-\mathrm{MHz}$ integration bandwidth when phase locking two Littrow-type diode lasers. This clearly shows the advantage of the GEECDL system over grating stabilized diode lasers with respect to reliable, lownoise phase locking.

We next phase lock GEECDL II to the fs-frequency comb and measure the phase noise LSD. The result of this meas-

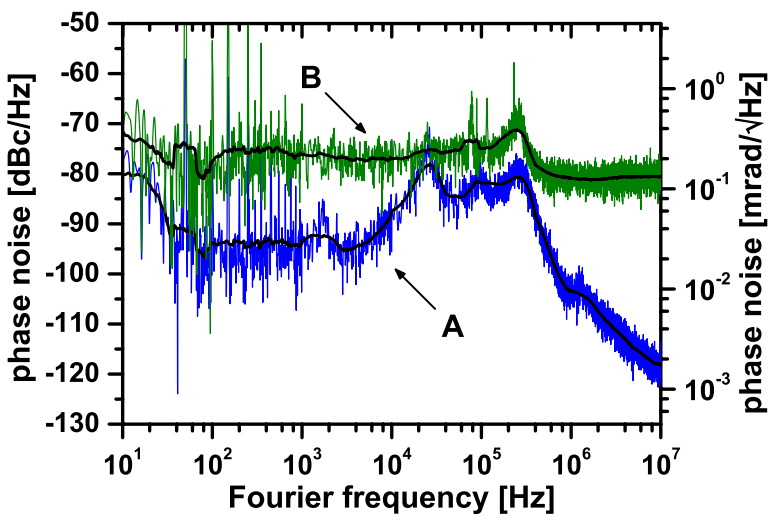

FIGURE 8 Single-sideband phase noise LSD of the rf signal derived from a beat-note measurement between GEECDL II and another laser. GEECDL II is either phase locked to GEECDL I (A) or phase locked to a fs-frequency comb (B). In the latter case the measurement sensitivity is limited by shot noise of GEECDL II: the coherent signal shown in Fig. 7 was recorded with a resolution bandwidth of $9.1 \mathrm{kHz}$, and hence the coherent signal power was $-28 \mathrm{dBm}$. Together with the shot-noise floor of $-111 \mathrm{dBm} / \mathrm{Hz}$ (graph (A) of Fig. 7), we estimate a shot-noise floor of $-80 \mathrm{dBc} / \mathrm{Hz}$ for the singlesideband phase noise shown as graph (B) above

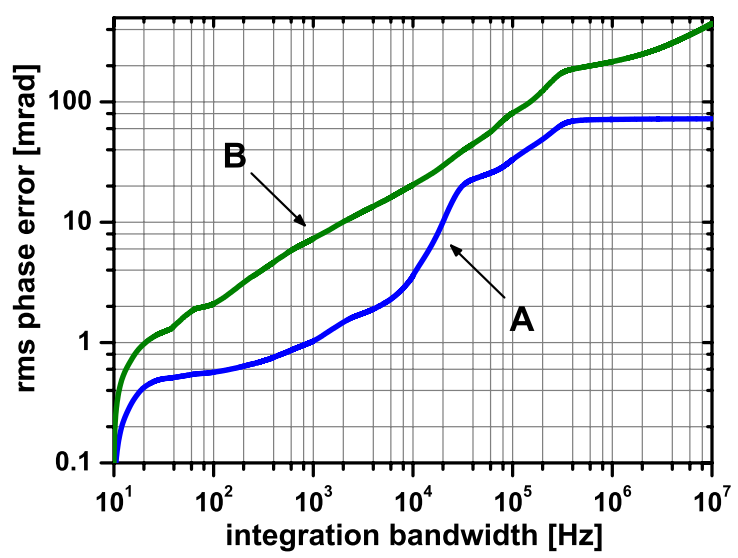

FIGURE 9 The rms phase error vs. integration bandwidth calculated from the single-sideband phase noise LSD shown in Fig. 8. Graphs (A) and (B) refer to phase locking two GEECDLs and phase locking GEECDL II to the fs-frequency comb, respectively

urement is shown as graph (B) in Fig. 8. The spectrum is flat from $10 \mathrm{~Hz}$ up to $250 \mathrm{kHz}$ where the OPLL servo bump appears. The measurement sensitivity is basically limited by shot noise from GEECDL II. Due to this noise floor, the rms phase error diverges for large integration bandwidths; see Fig. 9. A lower bound to the residual rms phase noise can therefore be derived by integrating the phase noise up to the $-3 \mathrm{~dB}$ corner frequency of the closed-loop OPLL transfer function, which is about $500 \mathrm{kHz}$. Integration of the noise up to $500 \mathrm{kHz}$ therefore yields a lower bound for the rms phase error of $\varphi_{\mathrm{rms}}=194 \mathrm{mrad}$. An upper, and clearly overestimating, bound of $\varphi_{\mathrm{rms}}=448 \mathrm{mrad}$ is derived by integrating the noise spectrum over the full $10-\mathrm{Hz}$ to $10-\mathrm{MHz}$ noise bandwidth. The fractional power contained in the carrier hence amounts to $96.3 \%$ and $81.8 \%$ for the lower and upper bounds, respectively. This agrees reasonably well with the fractional power of $96.3 \%$ and $86.8 \%$ derived earlier from the rf beatnote spectrum; see Fig. 7. Again, this is a remarkable result: Beverini et al. [6] required a control bandwidth of $1.2 \mathrm{MHz}$ to achieve a fractional power of $75 \%$ to be contained in the 
carrier, which corresponds to a residual rms phase error of $\varphi_{\mathrm{rms}}=536 \mathrm{mrad}$ according to (3).

The fundamental limitation that is imposed by shot noise or other types of detection noise on the noise reduction performance of the OPLL emphasizes the strong points of the GEECDL concept: as a relatively small servo bandwidth is sufficient to phase lock a GEECDL to a stable optical reference, only the (shot) noise within this relatively small bandwidth is fed into the system, and hence (fundamental) detection noise is kept at a minimum.

\subsection{Allan deviation}

To demonstrate the long-term stability of the phase lock between two GEECDLs and between a GEECDL and the fs-frequency comb we record a time series of instantaneous beat-note frequencies with a dead-time-free counter (MenloSystems) at 1-s gate time. The counter signal is derived from the photodetector in Fig. 2, which was earlier used to analyze a rf beat-note signal with a spectrum analyzer. Due to technical limitations of the counter we have to mix down the photodetector signal to approximately $45 \mathrm{MHz}$. The mixer output signal is then actively filtered by a tracking oscillator with a servo bandwidth of $940 \mathrm{kHz}$ before it is delivered to the counter. From the time series of tracking oscillator frequencies the Allan deviation is calculated and depicted in Fig. 10. When GEECDL II is phase locked to GEECDL I the beatnote signal shows a relative instability of $1.8 \times 10^{-16}$ at 1 -s integration time. For a phase lock between GEECDL II and the fs-frequency comb we achieve a relative instability of $5.4 \times 10^{-16}$ at 1 -s integration time. This stability is sufficient for coherent molecular spectroscopy with sub- $\mathrm{Hz}$ absolute accuracy.

\section{Conclusion}

We described the latest advancement of the GEECDL concept. This concept merges the well-established concepts of the grating stabilized diode laser and of the diode

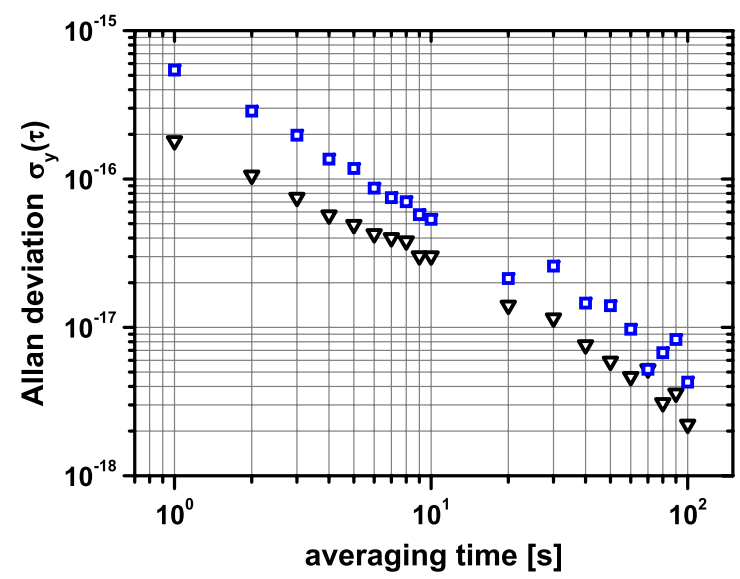

FIGURE 10 Standard Allan deviation of the beat-note signal between GEECDL II and an optical reference. GEECDL II is phase locked to GEECDL I (black triangles) and to the fs-frequency comb (blue squares). The larger Allan deviation of the lock to the fs-frequency comb reflects the fact that the fs-frequency comb's short-term frequency instability exceeds that of the GEECDL. No cycle slips were detected at the tracking oscillator output during the measurement for either of the traces laser with resonant optical feedback, thus combining large tuning range and reliable operation with excellent short-term frequency stability.

We reported the modification of the recent GEECDL resonator geometry. This modification provided a 10-fold enhancement of the output power, so that together with a 10 -fold more powerful laser diode chip the overall output power of the latest GEECDL version exceeds that of the earlier version by two orders of magnitude. The latest system now delivers $>100 \mathrm{~mW}$ of optical power at $1420 \mathrm{~nm}$.

The analysis showed that the GEECDL features a 10-fold reduction of frequency noise linear spectral density with respect to standard grating stabilized diode lasers. It was further demonstrated that this allows for phase stabilization of a GEECDL to a similar laser and to a fs-frequency comb with a simple, analog OPLL that employs a loop bandwidth as small as $237 \mathrm{kHz}$. The phase lock between two GEECDLs yielded a rms phase error of $72 \mathrm{mrad}$, and the fractional power contained in the coherent part of the beat-note signal corresponded to $99.48 \%$. The phase lock of the GEECDL to the fs-frequency comb revealed lower and upper bounds to the rms phase error of $194 \mathrm{mrad}$ and $448 \mathrm{mrad}$, respectively, corresponding to a fractional power of $96.3 \%$ to $82 \%$ contained in the carrier. We also demonstrated that the GEECDLs can phase-coherently follow a stable optical reference with an instability as small as $1.8 \times 10^{-16}$ at $1 \mathrm{~s}$.

These results equal or exceed the performance of the comparable grating stabilized diode laser system, however at a 10fold smaller servo bandwidth. It is important to emphasize that this performance could be improved even further, because the external feedback cavities used by us so far only had a modest finesse of 38 . We expect that a broadband reduction of the frequency noise linear spectral density by another order of magnitude is feasible, so that it should be possible to phase lock GEECDLs with a servo bandwidth that covers only the acoustic range of Fourier frequencies. GEECDLs are therefore the optimum choice when diode lasers have to be phase locked to a low-power optical reference in a high-noise environment, e.g. to a specific comb line of a fs-frequency comb. Because of their excellent passive short-term frequency stability we also consider the GEECDLs for the implementation of local oscillators for optical clocks.

ACKNOWLEDGEMENTS This work was supported by the Deutsche Forschungsgemeinschaft. We would like to thank Sacher Lasertechnik for technological support.

\section{REFERENCES}

1 R. Yamazaki, T. Iwai, K. Toyoda, S. Urabe, Opt. Lett. 32, 2085 (2007)

2 Q. Quraishi, M. Griebel, T. Kleine-Ostmann, R. Bratschitsch, Opt. Lett. 30, 3231 (2005)

3 T.W. Hänsch, R. Holzwarth, J. Reichert, T. Udem, in Proc. International School of Physics 'Enrico Fermi', Course CXLVI, ed. by T.J. Quinn, S. Leschiutta, P. Tavella (IOS Press, Amsterdam, 2001), pp. 747-764

4 A.D. Ludlow, X. Huang, M. Notcutt, T. Zanon-Willette, S.M. Foreman, M.M. Boyd, S. Blatt, J. Ye, Opt. Lett. 32, 641 (2007)

5 L. Cacciapuoti, M. de Angelis, M. Fattori, G. Lamporesi, T. Petelski, M. Prevedelli, J. Stuhler, G.M. Tino, Rev. Sci. Instrum. 76, 053111 (2005)

6 N. Beverini, M. Prevedelli, F. Sorrentino, B. Nyushkov, A. Ruffini, Quantum Electron. 34, 559 (2004)

7 F. Ducos, J. Honthaas, O. Acef, Eur. Phys. J. Appl. Phys. 20, 227 (2002)

8 M. Prevedelli, T. Freegarde, T.W. Hänsch, Appl. Phys. B 60, S241 (1995) 
9 A. Wicht, M. Rudolf, P. Huke, R.-H. Rinkleff, K. Danzmann, Appl. Phys. B 78, 137 (2004)

10 K. Döringshoff, I. Ernsting, R.-H. Rinkleff, S. Schiller, A. Wicht, Opt Lett. 32, 2876 (2007)

11 T.W. Hänsch, B. Couillaud, Opt. Commun. 35, 441 (1980)

12 S. Stry, S. Thelen, J. Sacher, D. Halmer, P. Hering, M. Mürtz, Appl. Phys. B 85, 365 (2006)
13 N. Strauß, I. Ernsting, S. Schiller, A. Wicht, P. Huke, R.-H. Rinkleff, Appl. Phys. B 88, 21 (2007)

14 L.D. Turner, K.P. Weber, C.J. Hawthorn, R.E. Scholten, Opt. Commun. 201, 391 (2002)

15 H. Loh, Y.-J. Lin, I. Teper, M. Cetina, J. Simon, J.K. Thompson, V. Vuletić, Appl. Opt. 45, 9191 (2006)

16 H.R. Telle, B. Lipphardt, J. Stenger, Appl. Phys. B 74, 1 (2002) 